\title{
Bending Contours in Silver Nanoprisms
}

Benito Rodríguez-González, Isabel Pastoriza-Santos, and Luis M. Liz-Marzán ${ }^{*}$

Departamento de Química Física, Universidade de Vigo, 36310, Vigo, Spain

e-mail: jbenito@uvigo.es; lmarzan@uvigo.es

\section{SUPPORTING INFORMATION}

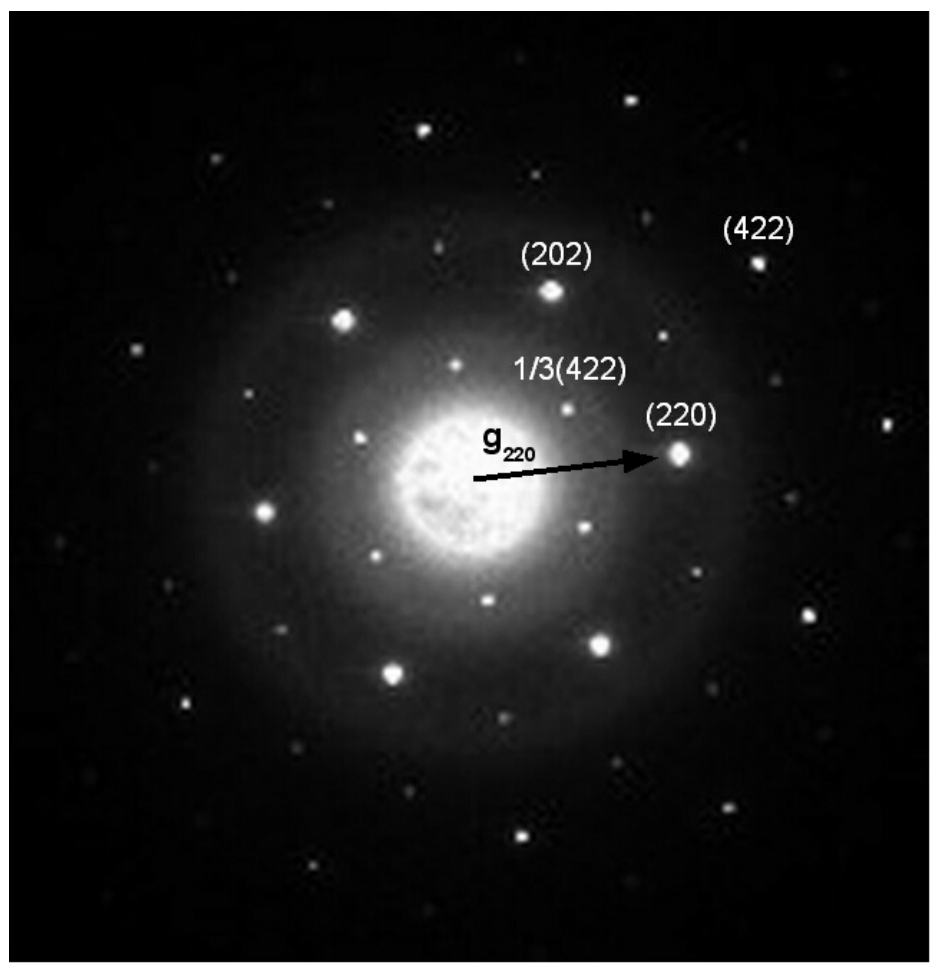

Figure S1. Selected area electron diffraction (SAED) pattern from a silver nanoprism, corresponding to a [111] zone axis. Indexed spots and the $\mathbf{g}_{220}$ diffraction vector are labeled. 

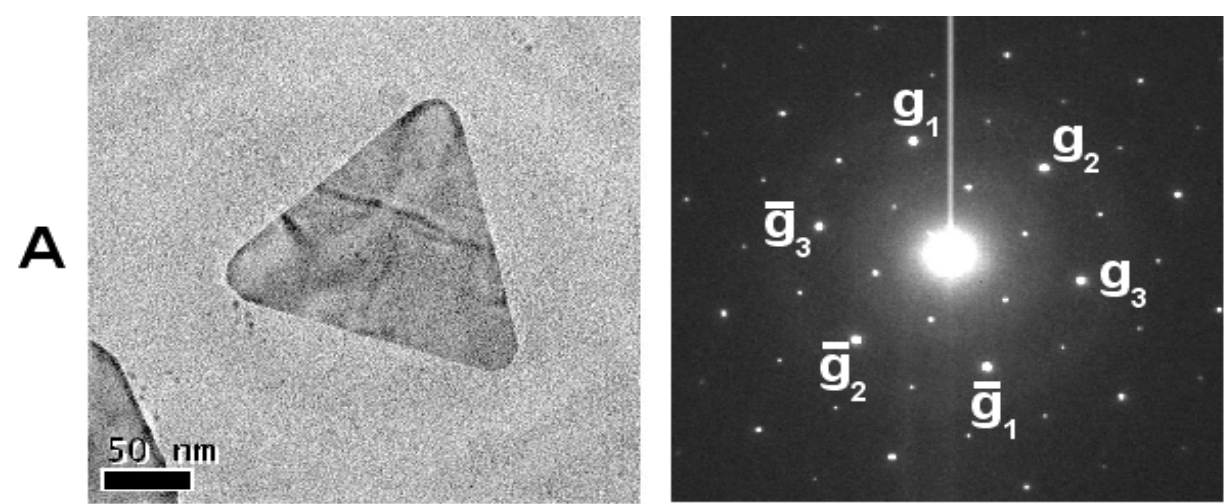

B
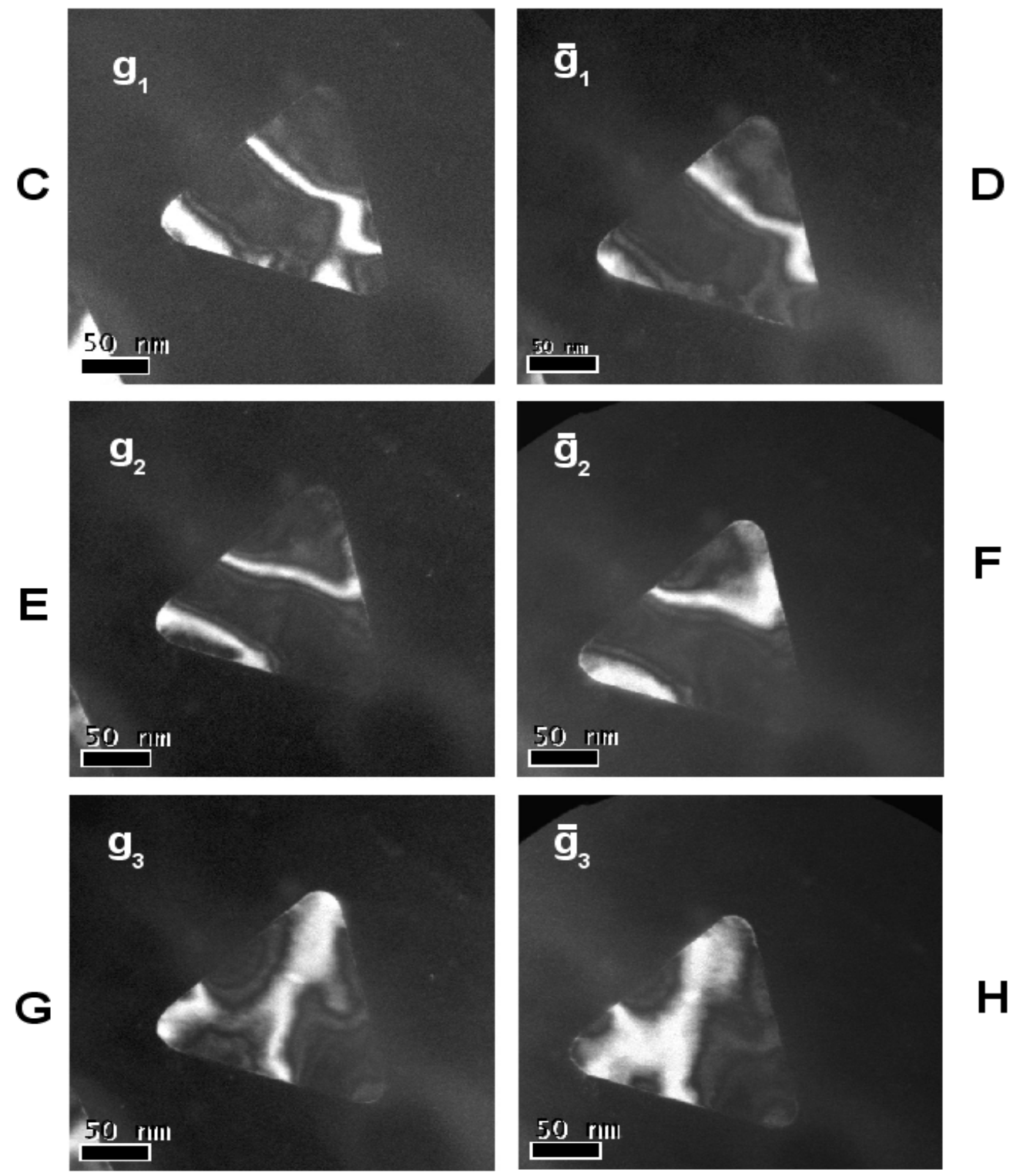

Figure S2. Dark field analysis of bending contours. (A) Bright field image of the selected nanoprism. (B) Corresponding SAED diffraction pattern. (C-H) Dark field images obtained by shifting the objective aperture. The diffraction vectors of the corresponding spots in B are indicated. The nanoprism shows a few bending contours, 
broad diffuse fringes and a well-defined bending contour across the entire particle. In the dark field analysis we observe that this bending contour does not correspond precisely with the crystalline planes associated to the spots with diffraction vectors $\mathbf{g}_{1}$ and $\mathbf{g}_{2}$, meaning that the bending edge is not located exactly on the direction of the corresponding planes. From the dark field images obtained using $\mathbf{g}_{\mathbf{1}}$ and $\mathbf{g}_{\mathbf{2}}$ spots we see that the bending contours are rather thin, as a result of a sharp bending that makes Bragg's law to be fulfilled in a thin area of the crystal. Oppositely, the dark field image obtained using the diffraction vector $\mathbf{g}_{3}$ yields areas with thicker fringes, arising from Bragg's law fulfillment in larger areas and thus pointing toward less sharp bending. We should however indicate that the curvature is never likely to be very pronounced, since the nanoprisms are always found to be within the zone axis. 